\title{
Developing a LTE Localization Framework using Real Network Data towards RAN Optimization through Context Knowledge
}

\author{
R. Borralho* \\ *Institute for Communication Systems \\ 5G Innovation Centre \\ University of Surrey \\ Guildford, United Kingdom \\ r.a.borralho@surrey.ac.uk
}

\author{
D. Duarte $\| \S$ \\ $\|_{\text {CELFINET }}$ \\ Consultoria em Telecomunicações, Lda. \\ Lisbon, Portugal \\ david.duarte@celfinet.com
}

\author{
A. Quddus* and P. Vieira ${ }^{\ddagger \S}$ \\ $\ddagger$ Instituto Superior de Engenharia de Lisboa \\ $\S$ Instituto de Telecomunicações \\ Lisbon, Portugal \\ a.quddus@surrey.ac.uk, \\ pedro.vieira@isel.pt
}

\begin{abstract}
The exponential growth of the network elements and data traffic exchange in the last few years elevated the need of network providers for optimized and cost-efficient solutions regarding network management and monitorization. Solutions such as drive-tests (DTs) are becoming extremely expensive with the vast extension and complexity of nowadays mobile networks. Therefore, this paper provides a solution for optimized networkcontext knowledge acquisition, towards the self-organizing networks (SONs) concept. The presented framework incorporates an entire scheme for network Traces processing and positioning, based on network measurements and fingerprinting techniques. This framework enables a series of different use cases for network management and optimization, with real-time data processing capabilities within the network Traces collection interval (15 minutes), and achieving a median positioning error of $90 \mathrm{~m}$.

Index Terms-Cellular networks, Minimization of Drive Test (MDT), network context, network Traces, Radio Network Planning and Optimization, self-organizing network, Context-Aware Networks.
\end{abstract}

\section{INTRODUCTION}

Nowadays, mobile networks represent one of the most innovative and challenging technology fields of work. The significant growth of mobile users together with the exponential increase in exchanged mobile data traffic resulted in demanding requirements for the network providers. These requirements are mostly verified through Quality of Service (QoS) and Quality of Experience (QoE) metrics. This assessment can be performed whether it is through the analysis of Configuration Management (CM), Performance Management (PM), using Key Performance Indicators (KPI), or even Drivetest (DT) data.

However, with network evolution and densification, these data sets are becoming more complex to handle and additional tools, methods, and data sources might be required to accomplish the performance objectives of legacy and future mobile communication technologies. In addition, methods such as DTs keep to become considerably expensive, with impracticable Operation Expenditure (OPEX) if the entire network needs to be monitored. This is caused due to the need for vehicles, human resources, and skilled measuring tools together with the amount of time (and resources) to cover the vast size of the mobile networks.

In this direction, network Traces are considered as a powerful data source for monitorization, management, and optimization of telecommunication networks. Network Traces encompass a significant part of the layer 3 exchanged signaling between the User Equipment (UE) and the network. A proper analysis of this data source allows to have a closer view of the network performance, with available information regarding the type of service and respective conditions experienced by the end-users. As a result, powerful outputs similar to those from DTs can be generated more efficiently.

The work explores the capabilities of a solution merging both Traces information and a Long Term Evolution (LTE) Geopositioning algorithm. Such a task involves the study of LTE signaling for the identification of crucial fields that can be used by the Geopositioning algorithm, to turn this data source into geo-referenced data. The development of a Geolocation algorithm based on fingerprinting techniques and Timing Advance (TA) data is part of this work. The output of this framework is expected to provide UE and network context information. Consequently, important benefits in terms of effectiveness and efficiency when managing and optimizing real networks can be achieved.

This article is structured as follows. Section II provides an overview of the crucial concepts for the understanding of the entire process and its achievements. In Section III, the main procedures regarding the framework implementation are described, focusing on the identification of crucial network events, the applied fingerprinting method for positioning, as well as its calibration. Section IV presents the outputs of this work along with providing a measure of the positioning error. Finally, Section V concludes the paper.

\section{BACKGROUND}

Several works within the literature have studied the Geopositioning process based on fingerprinting techniques and TA data [1], [2]. However, most of those studies are focused on 


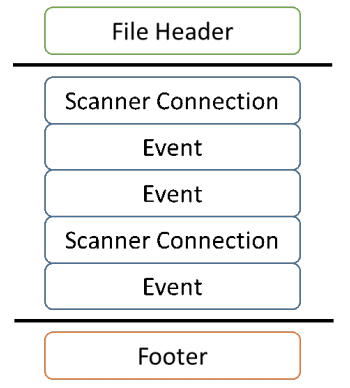

Fig. 1: Cell Trace Structure (adapted from [4]).

theoretical analysis and simulation environments. When moving to real network data, additional challenges and complexity exist. This depends on the available terrain data resolution, the real network data interpretation and processing, or the accuracy of Radiofrequency (RF) models. Therefore, and under this work, a study of a Geopositioning framework totally based on real network data is performed, applying a calibration model and TA filtering for maximizing positioning accuracy. The current section goes through the main concepts and details behind the framework study.

\section{A. The Ericsson's Cell Trace}

Similar to what happens for the Third Generation (3G) networks [3] with the Ericsson GPEHs, the Cell Trace is a file in the original format (.bin) collected directly from the mobile operator Operations Support System (OSS), i.e., from the live network. This file contains all the signalling exchanged between the end-users and the network. More specifically, between the users and a specific LTE Base Station (BS), during a period of 15 minutes. Typically, a dense urban LTE BS is composed by 3 cells in each frequency band [L800, L1800, L2600] MHz, with different defined antenna orientations (azimuths). While in $3 \mathrm{G}$ the GPEHs were collected at the Radio Network Controller (RNC) level, each LTE Cell Trace contains signalling information of one BS encompassing all its cells. The structure of a typical Cell Trace is illustrated in Figure 1. To understand this structure and format is of crucial importance to decode its information. The Header contains information about the scope of the recording; the scanners state (activated/deactivated) are reported in the Scanner Connection field; the major part of the file lies in the Event sections with all the signalling information; the Footer reports the time of normal termination of the file [4].

\section{B. The Timing Advance Parameter}

The TA parameter is specified by the Third Generation Partnership Project (3GPP) for the control of Uplink (UL) signal transmission timing [5]. The LTE BS (eNodeB) keeps measuring the time difference between the UL channels reception and the subframe time. Then, it can send a TA command to the UE, in order to change the UL transmission, aligning it with the subframe timing at the network side. The TA command indicates the change of the UL timing relative to the current UL timing in multiples of $16 \times T_{s}$, where $T_{s}$ is an LTE standard unit of time used across several specifications [6]. It is expressed as:

$$
T_{s}=\frac{1}{15000 \times 2048}[s]
$$

where 15000 represents the $15 \mathrm{kHz}$ sub-carrier spacing and 2048 is the maximum Fast Fourier Transform (FFT) size. There are two main LTE commands related with the TA parameter. It can be sent in the Random Access Response command from the Medium Access Control (MAC) layer, containing 11 bits. This is the initial TA estimation based on the Physical Random Access Channel (PRACH) requested by the UE. In this mode, the TA value index varies from 0 to 1282 and it is used for the calculation of the timing offset parameter $N_{T A}$. This parameter represents the offset between UL and Downlink (DL) radio frames at the UE, and is given by:

$$
N_{T A}=16 \times T_{A} .
$$

When used in the MAC Control Element command, the TA only has 6 bits, varying from index 0 to index 63 , and is used for adjustment of the previously defined offset values. In this scenario, the TA affects the timing offset according to:

$$
N_{T A, \text { new }}=N_{T A, \text { old }}+\left(T_{A}-31\right) \times 16
$$

where $N_{T A, \text { new }}$ and $N_{T A, \text { old }}$ indicate the new and previous timing adjustments, respectively.

\section{Minimization of Drive Test (MDT)}

Within the main data sources used for network management and optimization, DTs data is the only that contains detailed and accurate location information. However, this data source is limited to outdoor environments and to the routes where measurements were performed. In order to update a certain measurement and to check any changes with a previously tested environment, a new DT needs to be carried out. Therefore, this method consumes considerable amount of time and human effort, resulting in a costly and not efficient procedure. Driven by these challenges, 3GPP created a standardization named Minimization of Drive Tests (MDT) [7]. Consequently, several features were defined to mitigate the need for extensive DT campaigns. Within those features, it is highlighted to provide some location information capabilities to the UE, enabling the possibility of storing $\log$ files, and reusing radio measurements. In addition, this information is mostly available on network records, i.e., network Traces. In the following section, the main stages of the developed framework as well as the steps for its optimization within the desired objectives are presented.

\section{Framework Structure}

\section{A. Study and Identification of Relevant Network Traces}

Regarding the LTE network, all Cell Trace events contain common types of information, like the event name and identification, the timestamp of its recording, user identification in 
the specific area, as well as the serving cell details. However, all of them also contain specific information characterizing its function. With the information mentioned in the previous section regarding the Cell Trace characteristics, a parser portal that allows the decoding of the source .bin file was developed. It is possible to map the Event ID extracted from the decoding, and the Event Name available in the specific vendor documentation. The fields that constitute the event can also be decoded with this Cell Trace parser. Since this tool operates at the raw file level, it does not depend of any operator or intermediate parsers, which many times places a bottleneck for these processes.

Despite most network events follow the 3GPP standards, network real data always presents some light changes in structure and format depending on each vendor. Therefore, in order to enable the LTE outdoor location module, some useful events needed to be identified and studied, not only at the standards level, but also for the specific structure of the vendor data. This process resulted in the identification of three main events from the Cell Trace information [8]:

- RRC _MEASUREMENT_REPORT: This event processing allows to get parameters like Reference Signal Received Power (RSRP) and Reference Signal Received Quality (RSRQ), key measures of signal level and quality for LTE networks, and Physical Cell Identity (PCI).

- LTE_UE_MEAS INTRAFREQ1: This is an internal vendor network event similar to the RRC MEASUREMENT_REPORT. However, it filters the radio measurements for the same carrier. Such a characteristic can decrease the complexity of the framework processing which needs to be run per frequency.

- INTERNAL_PER_RADIO_UE_MEASUREMENT

_TA: This event represents UE level measurements with TA information sent periodically.

All the relevant decoded information is stored in suitable data structures to be used along the framework.

\section{B. Footprint Module}

The footprint module, where the BS cells coverage area is estimated, is based on typical cell fingerprinting techniques based on propagation modelling [9]. However, a calibration method is used to potentiate the accuracy of the coverage maps, combining the model with real network measurements. For each cell within the Region-of-Interest (ROI) where it is aimed to estimate the UEs position, a GeoTIFF [10] map for that area is loaded. This map can be considered as a matrix where each entry has a resolution of $30.3 \mathrm{~m}$ for latitude and $24.9 \mathrm{~m}$ for longitude. Therefore, for each $30.3 \times 24.9 \mathrm{~m}^{2}$ in the map, the parameters for the received power are calculated based on the cell configuration characteristics. The received power $P_{R}$ in a specific pixel $p$ served by a certain cell $c$, is given by:

$$
P_{R, p, c}=P_{T, c}+G_{T, p, c}+G_{R, p}-P_{L, p, c}
$$

where $P_{T, c}$ is the transmit power of cell $c, G_{T, p, c}$ is the transmitting gain of cell $c$ in pixel $p, G_{R, p}$ is the receiver gain at pixel $p$ and $P_{L, p, c}$ is the path loss on pixel $p$ taking into account the transmitting cell $c$.

The transmission gain $G_{T, p, c}$ is estimated based on 3GPP theoretical radiation pattern [11] as follows:

$$
G_{T, p, c}\left(\theta^{\prime \prime}, \phi^{\prime \prime}\right)=-\min \left\{-\left[A_{V}\left(\theta^{\prime \prime}\right)+A_{H}\left(\phi^{\prime \prime}\right)\right], G_{T, \max }\right\}
$$

where $G_{\text {Tmax }}$ is the transmission gain in the direction of the transmitter boresight, i.e., maximum directional gain of an antenna element, defined as $25 \mathrm{~dB}$. The $A_{V}\left(\theta^{\prime \prime}\right)$ and $A_{H}\left(\theta^{\prime \prime}\right)$ are the vertical and horizontal antenna attenuation, respectively, which are computed according to the following equations:

$$
\begin{gathered}
A_{V}\left(\theta^{\prime \prime}\right)=-\min \left[12\left(\frac{\theta^{\prime \prime}-90^{\circ}}{\theta_{3 d B}}\right)^{2}, S L A_{V}\right] \\
A_{H}\left(\phi^{\prime \prime}\right)=-\min \left[12\left(\frac{\phi^{\prime \prime}}{\phi_{3 d B}}\right)^{2}, A_{m}\right] .
\end{gathered}
$$

The $\theta_{3 d B}$ and $\phi_{3 d B}$ are the vertical and horizontal half power beamwidth apertures, respectively, and their standardized values are $10^{\circ}$ and $70^{\circ}$, respectively. The $S L A_{V}$ is the maximum vertical attenuation, and $A_{m}$ is the maximum horizontal attenuation, with typical values of $20 \mathrm{~dB}$ and 25 $\mathrm{dB}$, respectively [11].

For the path loss model, a combination of the COST-231 Hata and Lee model was used:

$$
P_{L, p, c}=\alpha_{1}+\alpha_{2} \log _{10}\left(h_{e f}\right)+\alpha_{3} \log _{10}\left(R_{p, c}\right)+\alpha_{4} L_{d i f, p}
$$

where $h_{\text {ef }}$ represents the BS effective height in $m, R$ is the distance between the pixel $p$ to the BS of cell $c$ in $\mathrm{km}$ given by:

$$
R_{p, c}=\sqrt[2]{\left(p_{l}-c_{l}\right)^{2}+\left(p_{j}-c_{j}\right)^{2}}
$$

where $l$ and $j$ represent the latitude and longitude coordinates, respectively. Additionally, $L_{\text {dif }}$ are the difraction losses calculated through the Deygout method limited to a maximum of 3 edges [12]. The independent factors $\alpha_{1}, \alpha_{2}, \alpha_{3}$, and $\alpha_{4}$ are specific parameters and related to the system frequency, effective height, distance, and diffraction losses, respectively.

1) Model Calibration: After calculating the cell footprints, a calibration process is applied to the results based on DT data, when available. Otherwise, typical values based on the existing propagation model for the path loss independent factors are provided [9]. When DTs data is available in the bins of interest, information of average received power is obtained, which allows to optimize path loss values based on DTs. In this direction, the path loss equation already mentioned parameters, $\alpha_{1}, \alpha_{2}, \alpha_{3}$ and $\alpha_{4}$, are calibrated and estimated through the linear least squares regression method. Using this method, the difference between the path loss model estimated values and the measurements provided by the DTs will be minimized. The calibrated model can then be extrapolated and applied in similar environments. 


\section{Positioning Module}

The positioning module makes use of the identified events from network Traces presented in section III-A and the previously calculated footprints. This process starts by using the information in the LTE_RRC _MEASUREMENT_REPORT and LTE _UE _MEAS _INTRAFREQ1 events, containing received power information for each of the cells measured by the UE. The positioning process is based on the minimization of the Mean Absolute Error (MAE) between the received power values reported in the Traces, and the generated footprints.

In this process, each measurement event needs to have more than one entry of received power, i.e., it should be the result of a network record with at least two reported cells. For each of those entries, a cell is associated with its respective footprint. The reported received power of each cell is subtracted to the received power of each bin of the corresponding cell footprint, generating a delta matrix:

$$
\Delta P_{R, r, c}=\left|P_{R \text { footprint }, r, c}-P_{R e}\right|
$$

where $\Delta P_{R, r, c}$ is the delta matrix entry with row $r$ and column $c, P_{R \text { footprint }, r, c}$ is the received power of the estimated footprint matrix on row $r$ and column $c$ entry, and $P_{R, e}$ is the received power entry $e$ in the network event under analysis. A delta matrix is calculated for each reported cell (entry) of the event under analysis. Afterwards, a superimposed footprint is computed for each event. This matrix results in overlapping the previously generated delta matrixes. The superimposed matrix (S) is derived as follows:

$$
S=\frac{\sum_{k=1}^{N} \Delta P_{R, k}}{N}
$$

where $k$ is the delta footprint from a report entry and $N$ is the total number of the event reported cells.

1) Event Potential Locations Filtering: In the superimposed matrix, the entries with lower values are considered the most probable locations of the event under analysis, since they represent the lower differences between the reported values from Traces and the generated footprints. Nonetheless, at this stage, several areas in the superimposed matrix are candidates for positioning the report. In order to mitigate this, additional filtering was used before the process of deciding the report final location. The first filter is based on the antenna gain pattern of the reported cells. Since the UEs are usually served by the cell that provides higher gain in a certain area, the radiation pattern will allow to select the bins in the superimposed matrix with higher antenna gain.

A second filtering process regards on the use of the TA attribute contained in the LTE _INTERNAL_PER _RADIO _UE _MEASUREMENT_TA event. As aforementioned, the TA value can be mapped into a distance measure to the serving cell. In LTE, this represents a distance of $78.12 \mathrm{~m}$ for each TA value. In this direction, the superimposed matrix is cropped into a smaller matrix, centered in the serving cell and with radius equal to the distance value mapped from the TA attribute. This will considerably narrow down the available locations for the report positioning.
2) Setting Event Final Location: After deriving the superimposed matrix and filtering some locations with the report cells radiation pattern and the TA information, a final position is attributed to the event under analysis. This process uses two methods: the minimum bin or center of mass. The minimum bin is a straight forward method, where the matrix entry with the lower value, i.e., with the lower MAE is considered as the user position. Although this is a good approach for the events reporting good values of received power, the same cannot be said when these values are low, since they cause increased uncertainty. In such a scenario, the center of mass is used.

The center of mass method basically consists in defining the report location as an averaging of the MAE values of the superimposed matrix. This method is applied to find a final row according to:

$$
\text { Row }=\text { Round }\left(\frac{\text { Sum }_{\text {row }}}{\text { Sum }_{\text {matrix }}}\right)
$$

where the Sum matrix $_{\text {is }}$ ise summation of all superimposed matrix values, and Sum $_{\text {row }}$ is computed as follows:

$$
\text { Sum }_{\text {row }}=\sum_{r=1}^{R}\left[\left(\sum_{c=1}^{C} S_{[r, c]}\right) \times r\right]
$$

where $R$ and $C$ are the number of rows and columns of the superimposed matrix $S$.

Analogously, the same process is performed for the final superimposed matrix column:

$$
\text { Col }=\text { Round }\left(\frac{\text { Sum }_{\text {col }}}{\text { Sum }_{\text {matrix }}}\right)
$$

where $S u m_{c o l}$ is given by:

$$
\text { Sum }_{c o l}=\sum_{c=1}^{C}\left[\left(\sum_{r=1}^{R} S_{[r, c]}\right) \times c\right] .
$$

The final event location process is concluded after this step.

\section{RESUlTS}

The area under test for this algorithm evaluation is centered in a dense urban BS in the city of Lisbon, Portugal. Network Traces and DTs belonging to the cells from this BS were collected to produce the results presented in this section.

\section{A. Framework Produced Use Cases}

Several use cases can be achieved by understanding and processing the information contained in the network Traces. This information can be crucial for the management and monitorization of future mobile networks, without expending significant operational costs in the process. Figure 2 shows the RSRP mapping in the area of interest, based on traces information processing. Such use case allows to understand the areas were a specific cell or BS is serving UEs. Another interesting use case regarding network monitorization is related with the PCI mapping. In Figure 3, the processed network Traces are grouped by PCI and positioned with the 


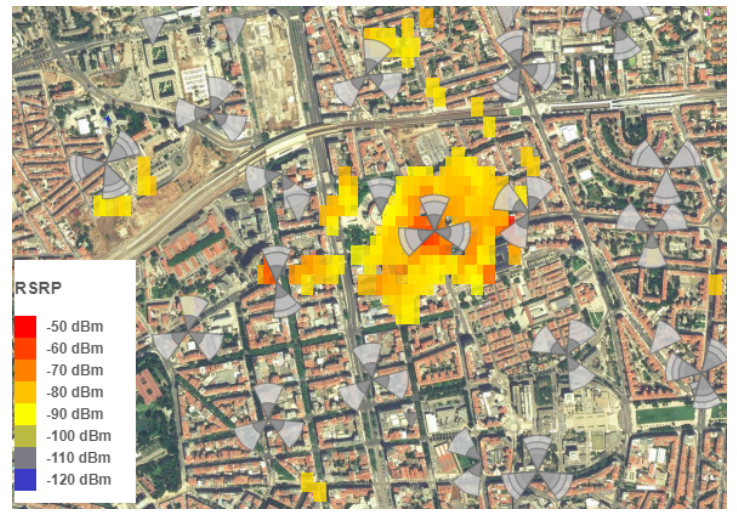

Fig. 2: RSRP Use Case Thematic Map.

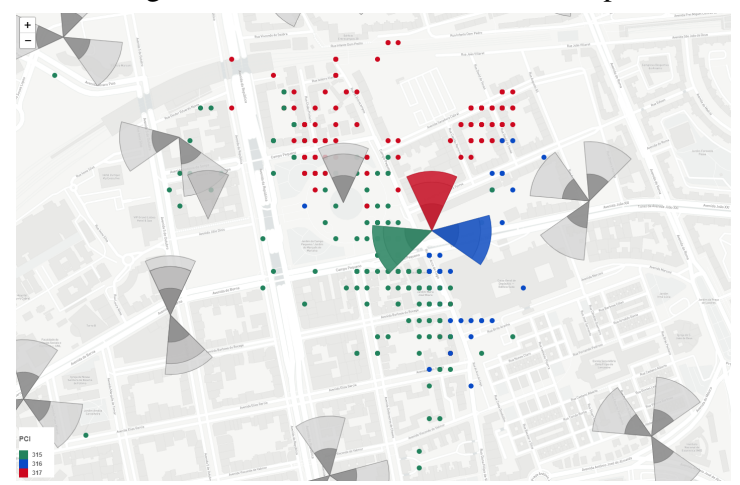

Fig. 3: PCI Use Case Thematic Map.

Geolocation algorithm, being associated to the respective BS sector. The result in Figure 3 not only shows this powerful use case, but it also validates the algorithm processing, since most of the Traces attributed locations are in accordance with the reported PCIs. This use case can also be used to detect PCI crossed sectors.

The aforementioned use cases sample how the network Traces processing can overcome the DTs, providing similar results with much lower costs for service providers. Further framework analysis shows how the Traces positioning error is related with the corresponding DTs. In Figure 4, the solid circles represent the positioned Traces, while the crosses represent the respective DTs. The green color represents the lowest errors (below $100 \mathrm{~m}$ ), yellow represent errors between 100 $\mathrm{m}$ and $200 \mathrm{~m}$, and the red color represents errors above 200 $\mathrm{m}$. Despite the positioning error, a big correlation is verified between DTs and positioned network Traces. Not only it is possible to observe that Traces mostly follow the DTs location as the biggest errors are related with higher distances to the cell, or with Non-line-of-sight (NLOS) scenarios following higher levels of RSRP degradation.

\section{B. Error Analysis and Validation}

The positioning error analysis not only helps to know the actual positioning error, but it also allows to understand which sections from the cell covering area causes bigger disturbance in the positioning process.

Figure 5 presents the Probability Density Function (PDF) and Cumulative Distribution Function (CDF) positioning error distributions for the processed network Traces. It is again

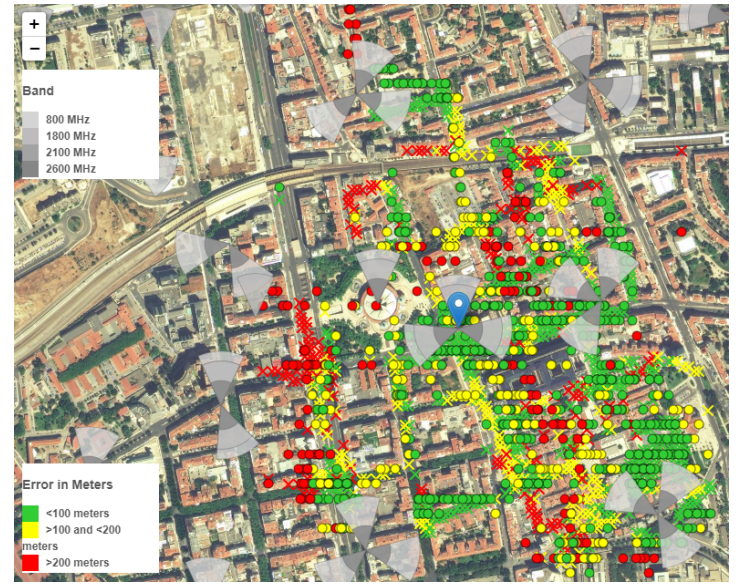

Fig. 4: Positioned Traces and respective DT error relation.

clear by analysing the PDF that most positioning errors lie below the $200 \mathrm{~m}$ mark. According to the $\mathrm{CDF}$, the positioning result errors considering the analysis of 15 minutes of layer 3 messages from network Traces are as shown in Table I. The analysis of these two processes shows that $95 \%$ of the positioned reports have an error below $320 \mathrm{~m}$. Furthermore, the median and average errors are $92 \mathrm{~m}$ and $115 \mathrm{~m}$, respectively. Considering the fact that real data was used for the entire process, with a previous identification and processing of significant amount of network Traces data, providing an almost real-time framework operation inside the 15 minutes of Traces collection granularity, is a very encouraging result. In fact, the positioning error is aligned or even in some scenarios is better than what is achieved in more controlled environments, as in [13].

Additionally, Figure 6 confirms the previously observed trend regarding the positioning error and the distance to the cell, with a correlation above $50 \%$. For farther distances to the cell, the RSRP tends to be lower and more significant interference appears due to the proximity with the cell-edge, which downgrades the algorithm accuracy. In a dense urban environment as the one under test, where cell range usually is lowe than $500 \mathrm{~m}$, if a shorter distance for positioning is considered, avoiding the cell-edge locations, positioning accuracy can be enhanced at least by $10 \%$.

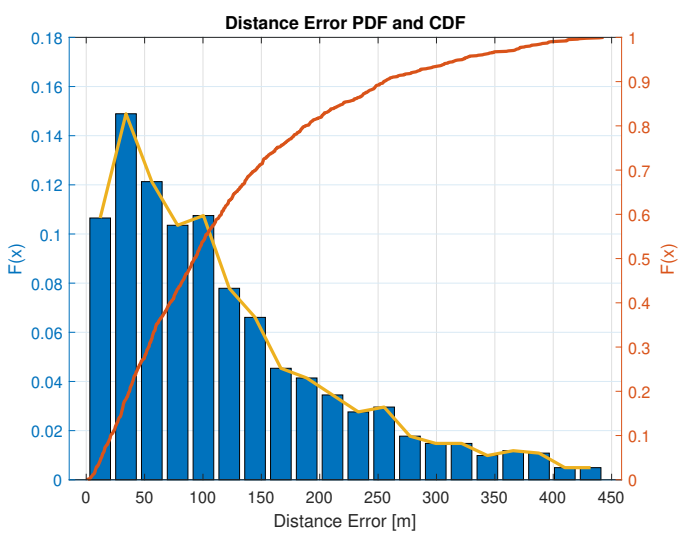

Fig. 5: Distance Error PDF (left y-axis) and CDF (right y-axis) Distributions. 
TABLE I: Positioning Error Metrics.

\begin{tabular}{c|c}
\hline Metric & Distance Error [m] \\
\hline 5th-Percentile & 13 \\
\hline Median & 92 \\
\hline 95th-Percentile & 322 \\
\hline Average & 115 \\
\hline
\end{tabular}

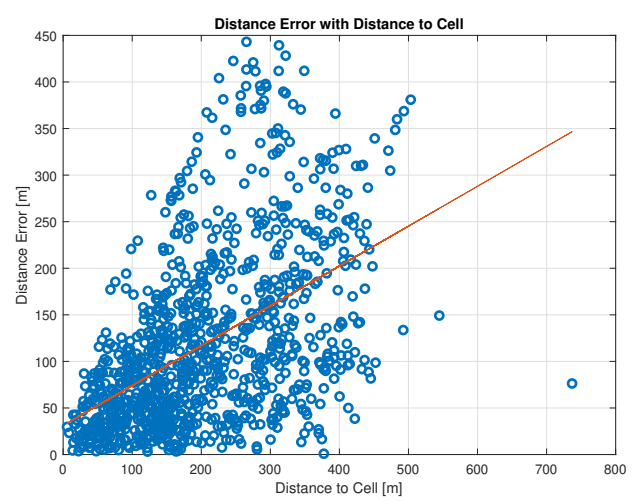

Fig. 6: Positioning Error Variation with Distance to the Cell.

Despite some existent higher complexity positioning algorithms may decrease the positioning error, this not always fits the mobile operator needs. This type of process encompasses considerable high amounts of data. Therefore, when merged with very complex Geopositioning algorithms, handling 15 minutes of signalling may result in much more processing time. For certain use cases, in order to understand the radio network behaviour in certain areas, it is not necessary to have very precise positioning. In this direction, using a simpler Geopositioning algorithm which provides good positioning errors can be a powerful solution for several network monitoring use cases, providing coherent and valid results of network/user-context, enabling near real-time processing and analysis.

\section{CONCLUSIONS}

This work presents a framework for network Traces processing and positioning towards Radio Access Network (RAN) optimization based on network and user-context knowledge. All this work is based on real network data. Most results on this area do not assume real network data in the entire process extension. Processing and positioning real network Traces bring additional challenges in terms of processing time and uncertainties due to the several different stages of the framework. In this paper, a deep study on network traces and signalling analysis was performed in order to understand how the UE reports several radio condition details to the BS. An entire structure for analysis, collection, and processing of this data was performed. In addition, a geopositioning algorithm based on fingerprinting techniques was produced. A least squares mathematical calibration for accurate BS coverage estimation based on the environment under test was performed. The positioning technique was complemented with TA information collected from network Traces.

The final results showed an entire optimized process for network context use cases production in almost real-time, inside the 15 minutes period of each communication set. Furthermore, an error analysis shows a median positioning error around $90 \mathrm{~m}$, which is a coherent result for the use cases within the scope of this paper. The entire process is scalable and can easily accommodate different layers of complexity, whether it is for the positioning process, calibration, or fingerprinting technique. Finally, this framework presents a base for DT minimization, studied and developed under real network parameters and patterns. Such a study can highly increase the operation cost savings for management and monitorization of future mobile networks.

\section{ACKNOWLEDGMENT}

The authors would like to thank "Instituto de Telecomunicaçoes" (IT) and CELFINET for financing the work developed in this paper, as well as the "Programa Operacional Competitividade e Internacionalização" (COMPETE) 2020 for supporting the project GARMIO (POCI-01-0247-FEDER-033479), financed by the "Fundo Europeu de Desenvolvimento Regional" (FEDER).

\section{REFERENCES}

[1] Z. Shakir et. al., "LTE Geolocation Based on Measurement Reports and Timing Advance," In: Arai K., Bhatia R. (eds) Advances in Information and Communication. FICC 2019. Lecture Notes in Networks and Systems, vol 70. Springer, Cham.

[2] L. Jarvis, J. McEachen and H. Loomis, "Geolocation of LTE subscriber stations based on the timing advance ranging parameter," 2011 MILCOM 2011 Military Communications Conference, Baltimore, MD, 2011, pp. 180-187.

[3] R. Borralho et. al., "A Novel Localization C\# Framework for RAN Optimization Using Extreme Programming Methodologies," 2017 20th International Symposium on Wireless Personal Multimedia Communications (WPMC)', Bali, 2017, pp. 516-521.

[4] Ericsson, Performance Events File Format - Description, 2017.

[5] 3GPP, "Technical Specification Group Radio Access Network; Evolved Universal Terrestrial Radio Access (E-UTRA); Physical layer procedures (Release 14), Technical Specification (TS) 36.213, 12 2019, version 14.3.0.

[6] 3GPP, "Technical Specification Group Radio Access Network; Evolved Universal Terrestrial Radio Access (E-UTRA); Physical channels and modulation (Release 15), Technical Specification (TS) 36.211, 022020 , version 15.9.0.

[7] W. A. Hapsari et al., "Minimization of drive tests solution in 3GPP," in IEEE Communications Magazine, vol. 50, no. 6, pp. 28-36, June 2012.

[8] G. Kasinathan, "Data Transformation Trajectories in Embedded Systems," Independent thesis Advanced level, KTH, School of Information and Communication Technology (ICT), 2016.

[9] M. S. Mollel and M. Kisangiri, "An overview of various propagation model for mobile communication," Proceedings of the 2nd Pan African International Conference on Science, Computing and Telecommunications (PACT 2014), Arusha, 2014, pp. 148-153.

[10] Copernicus, Europe's eyes on Earth, [Online]: https://land.copernicus.eu/ October (accessed in 06 2020).

[11] 3GPP, "Technical Specification Group Radio Access Network; Evolved Universal Terrestrial Radio Access (E-UTRA); Further advancements for E-UTRA physical layer aspects (Release 9), Technical Specification (TS) 36.814, 03 2017, version 9.2.0

[12] RECOMMENDATION ITU-R P.526-8, "Propagation by diffraction," International Telecommunication Union, Standard, Jan. 2018.

[13] T. Hiltunen, J. Turkka, R. Mondal and T. Ristaniemi, "Performance evaluation of LTE radio fingerprint positioning with timing advancing," 2015 10th International Conference on Information, Communications and Signal Processing (ICICS), Singapore, 2015, pp. 1-5. 